\title{
O papel do comitê de ética em pesquisa na avaliação de testes estatísticos
}

Wilson Paloschi Spiandorello

\section{Resumo}

A ética em pesquisa no Brasil tem passado por transformações desde que se estabeleceu a Plataforma Brasil, em 2011, e a Resolução CNS 466/12, em 2013. No entanto, as implicações éticas do uso de testes estatísticos na pesquisa quantitativa têm sido pouco discutidas. Alguns defendem que os comitês de ética em pesquisa não devem se envolver com análises estatísticas, ao passo que outros advogam o contrário. Por meio de levantamento na literatura, este artigo revisa algumas implicações éticas do uso de testes estatísticos e analisa as consequências de desvios estatísticos sobre os resultados finais dos estudos, defendendo que os comitês devem assumir responsabilidade quanto à adequação das análises estatísticas nos projetos avaliados. Diante das atuais dificuldades de estruturação do sistema de avaliação da ética em pesquisa no Brasil, propõe-se aqui orientações básicas para a avaliação da análise estatística, apontando solução para esse impasse por meio da pesquisa reproduzível.

Palavras-chave: Ética em pesquisa. Estatística e dados numéricos. Pesquisa. Métodos.

\section{Resumen}

\section{El papel del comité de ética en investigación en la evaluación de pruebas estadísticas}

Brasil ha pasado por transformaciones en el campo de la ética en las investigaciones, desde que fue creada la Plataforma Brasil, en 2011, y la Resolución CNS 466/12, en 2013. Sin embargo, las implicaciones éticas del uso de las pruebas estadísticas en la investigación cuantitativa se han discutido muy poco. Mientras que algunos defienden que los comités de ética en investigación no deben estar involucrados en análisis estadísticos, otros dicen lo contrario. Este artículo examina con el análisis de la literatura, el papel de las implicaciones éticas del uso de pruebas estadísticas y analiza las consecuencias de las desviaciones estadísticas sobre los resultados finales de los estudios y se llega a la conclusión de que los comités deberían asumir la responsabilidad de la adecuación de los análisis estadísticos en los proyectos evaluados. Frente a las dificultades de estructuración del sistema de evaluación de la ética en investigación en Brasil, se propone aquí orientaciones básicas para la evaluación de los análisis estadísticos, destacando una solución para este impase por medio de la investigación reproducible.

Palabras-clave: Ética en investigación. Estadística y dados numéricos. Investigación. Métodos.

\section{Abstract}

The role of the committee on ethics in research in the assessment of statistical tests

Brazilian's research ethics has being transformed since the deployments of Platform Brazil, in 2011, and the Resolution CNS 466/12, in 2013. However, the ethical implication of statistical tests uses in quantitative research has been little discussed. While some advocate that the research ethics committees should not be involved with statistical analyzes, others argue the opposite. This paper reviews the role of the ethical implications of the statistical work of research, analyzes the consequences of statistical deviations on the final results of the work and concludes that the committee should take responsibility for the adequacy of the statistical analyzes in research projects. Facing current difficulties regarding establishing an ethic assessment system in research in Brazil, some basic orientation is proposed to statistical analyzes assessment, pointing out a solution to this issue by the so called reproducible research.

Keywords: Research, ethics. Statistics and numerical data. Research. Methods.

Doutor wilsonsp@terra.com.br - Hospital Geral, Caxias do Sul/RS, Brasil.

Correspondência

Rua Lionilda Fassoli Zatti 201 casa 4, Jardelino Ramos CEP 95050-250. Caxias do Sul/RS, Brasil.

Declara não haver conflitos de interesse. 
Este trabalho é fruto da reflexão do autor, que ao trabalhar com a pesquisa acadêmica encontrou divergência de opiniões quanto ao papel dos comitês de ética em pesquisa (CEP) na análise estatística dos projetos submetidos à avaliação institucional. Na própria literatura, enquanto alguns defendem a ideia de que essa atribuição é unicamente do pesquisador, outros propõem que as análises estatísticas atinentes aos projetos devem ser realizadas pelos CEP, uma vez que o desenho da pesquisa e a metodologia aplicada se originam de estatísticas ${ }^{1}$. Da mesma forma, tanto os resultados quanto as conclusões do estudo decorrem de achados estatísticos utilizados para aceitação ou rejeição da proposta teórica, bem como para a tomada de decisões que podem beneficiar ou não o ser humano.

Se o papel da estatística no delineamento de um projeto quantitativo transparece à primeira vista, é patente também a relação entre ética e projeto de pesquisa, pois no Brasil, assim como em vários outros países, se considera que uma pesquisa científica em seres humanos seguiu padrões éticos adequados quando o projeto que the deu origem foi avaliado e aprovado por um CEP competente e independente.

Entretanto, muito embora pareça evidente a relação do projeto de pesquisa com a ética e a estatística, levantamento realizado em outubro de 2013, na Medline, registrou somente 26 artigos cujo título contém os vocábulos "statistics" e "ethics", oito deles do mesmo autor, Douglas G Altman ${ }^{2-9}$, produzidos nos anos de 1980 e 1981 . Esses artigos abordam a obtenção dos dados, análise, delineamentos de pesquisas, tamanho da amostra, interpretação dos resultados, apresentações dos resultados, mau uso da estatística, qualidade dos trabalhos publicados em revistas médicas. Dentre os outros 18 artigos ${ }^{10-27}$ associando os dois temas naquela base de dados, apenas seis foram publicados a partir do ano $2000^{22-27}$. O pequeno número de artigos publicados, porém, não significa que ética e estatística se dissociaram, embora indique a necessidade de revisar atualmente o grau dessa relação.

Uma explicação para a diminuta frequência de trabalhos que contemplam os dois aspectos nos projetos de pesquisa quantitativos pode relacionarse com o fato de que os assuntos éticos discutidos por Altman hoje se acham incorporados como vieses à pesquisa. No trabalho intitulado "Basic of medical studies", Abhaya Indrayan ${ }^{28}$ aponta isso ao listar 36 vieses e as formas de evitá-los, as quais foram introduzidas no método de pesquisa. Desses métodos, salientamos alguns dos principais aspec- tos que supostamente devem estar presentes na relação entre ética e estatística na ciência. Assim, espera-se:

- da ciência a generalização, isto é, que os conhecimentos sejam verossímeis em populações semelhantes; que os resultados sejam reprodutíveis, e que seja possível diminuir os graus de incerteza das predições;

- que o projeto de pesquisa seja um documento completo que explicite a priori o método científico aplicado na pesquisa e como ela será operacionalizada, e, dessa forma, que o projeto constitua um roteiro a ser rigorosamente seguido do começo ao fim;

- que a análise estatística seja fiel ao teste de hipótese ou aos objetivos descritos no projeto, de modo que os dados não sejam dirigidos consciente ou inconscientemente para um resultado em especial;

- que o trabalho escrito resultante da pesquisa tenha respeitabilidade e credibilidade, isto é, o leitor precisa partir do pressuposto de que tudo foi feito de forma correta, de acordo com os limites da ciência, e que a aplicação do conhecimento resultante do estudo beneficiará ou, no mínimo, não causará mal ao ser humano. Somente é ético realizar experimentos científicos bem formatados com o método científico ${ }^{8}$.

Além desses aspectos gerais, no Brasil as pesquisas médicas devem seguir também as normas e diretrizes do sistema CEP/Conep. Como é de conhecimento geral, a regulamentação ética da pesquisa no Brasil é chancelada pelo Conselho Nacional de Saúde (CNS), órgão colegiado adstrito ao Ministério da Saúde. Por intermédio do CNS, a Comissão Nacional de Ética em Pesquisa (Conep) e os CEP locais, distribuídos pelo país, aplicam as recomendações e pressupostos regulamentados pela Resolução CNS $466 / 12^{29}$ e suas relacionadas.

Em decorrência do cumprimento da resolução, espera-se dos CEP a proteção ética tanto dos participantes da pesquisa quanto da população que irá utilizar os conhecimentos advindos do estudo. Tal proteção ética pauta-se, entre outras coisas, pelo respeito à autonomia do participante, pela beneficência e a não maleficência do estudo no que concerne à saúde e integridade física do participante, bem como pela justiça na distribuição dos ônus e benefícios nas dimensões individual e coletiva. A proteção se estende ainda à avaliação da exposição a riscos desnecessários e à utilização dos recursos de forma produtiva, já que esses são finitos e muitas vezes escassos. 
Para contemplar todos esses aspectos éticos, os resultados dos estudos precisam ser cientificamente corretos. Um resultado cientificamente correto não implica ausência de erro, mas o erro somente se justifica quando é inerente à própria limitação do método científico, não devendo nunca decorrer de omissão ou negligência deliberadas. Sendo assim, espera-se que o CEP atue como fiador da respeitabilidade e da credibilidade da pesquisa e, por conseguinte, como instância de proteção dos participantes da pesquisa, deixando de aprovar projetos que não respondam cientificamente às questões da pesquisa. Acreditamos que essa atribuição justifica a análise da estatística dos projetos submetidos à avaliação pelos CEP, como buscaremos mostrar a seguir.

Pressupostos para aplicação dos testes estatísticos

A estatística é um componente da ciência ligado a modelos de pesquisas que, na área da saúde, são englobados pela epidemiologia. Seja na área da epidemiologia descritiva, que trata das ocorrências das doenças ou agravos à saúde, seja no âmbito da epidemiologia analítica, que trata de suas associações e relações casuais, haverá um delineamento de estudo que encaminha os resultados para serem analisados pela estatística. Os delineamentos podem ser classificados em observacionais (transversal, coorte ou caso-controle) ou intervencionistas (ensaios clínicos). É importante considerar que o desenho falho e a aplicação negligente de uma pesquisa quantitativa, especificamente na área da saúde, podem causar sérios danos a indivíduos e coletividade. Para uma revisão didática e sucinta dos diferentes desenhos de pesquisa, sugerimos a leitura do artigo de Hochman, Nahas, Oliveira Filho e Ferreira, Desenhos de pesquisa ${ }^{30}$.

Antes de discutir a pertinência da análise pelos CEP da aplicação dos parâmetros estatísticos das pesquisas submetidas à avaliação institucional, é necessário esclarecer o contexto no qual a estatística se insere. Conforme definição dicionarizada, estatística é a ciência de coletar, apresentar e analisar dados numéricos ${ }^{31}$. A análise estatística opera segundo a teoria da probabilidade, sendo utilizada em projetos de pesquisas quantitativas baseados em variáveis amostrais. Variáveis são os conjuntos das medições dos fenômenos em estudo. A possibilidade de generalizar as informações levantadas nos estudos decorre das características da amostra, que deve espelhar o aspecto da realidade que se pretende retratar.
Conceitua-se projeto de pesquisa o documento que descreve objetivamente, por meio do método científico, a questão da pesquisa e como essa questão será respondida. Projetos de pesquisa consistem em exposição do problema em tela, objetivos, método e resultados. Alguns tipos de projeto agregam ainda discussão e considerações finais, ou conclusão, conforme o caso. Nas pesquisas quantitativas, que buscam descrever certo aspecto dos fenômenos naturais mensuráveis, pode-se também formular uma hipótese e, mediante testes estatísticos, produzir evidências que a apoiem ou contradigam.

A hipótese é a proposta do pesquisador para a solução do problema da pesquisa. O teste de hipótese é a procura por evidências probabilísticas que levem a aceitar ou rejeitar a hipótese. Pela análise probabilística do conjunto desses elementos, os autores concluem sobre os objetivos explicitados. Desse modo, a estatística é instrumento analítico fundamental nos trabalhos científicos quantitativos. O mau uso da estatística, que produz resultados errôneos, inutiliza o trabalho científico; isso, portanto, pode ser caracterizado como questão ética ${ }^{4}$.

Como os testes estatísticos são fórmulas matemáticas desenvolvidas para calcular a probabilidade de ocorrência de determinados fenômenos em diversas circunstâncias, sempre há um modelo e uma teoria envolvidos nos resultados estatísticos. Quando os estudos são descritivos, a estatística infere parâmetros populacionais. Já os estudos analíticos são modelos destinados a verificar hipóteses que, na área da saúde, são geralmente avaliadas por parâmetros relacionados com a estatística vital, como demografia, natalidade, incidência de enfermidades e mortalidade na população ou subgrupo.

Para explicar os critérios de seleção desses parâmetros, podemos pensar na análise de intenção de votos, aplicada em épocas de eleições, pela qual se deseja conhecer o candidato com maior probabilidade de votos. Para tentar, de fato, inferir o resultado, necessita-se - obrigatoriamente - de amostra probabilística ${ }^{7}$, ou seja, um conjunto de pessoas que retrate as principais características da população, incorporando variáveis de sexo, etárias, educacionais, econômicas, bem como aquelas relativas a outros aspectos culturais e as associadas às condições de vida.

Para entender melhor como se define uma amostra probabilística, é necessário conhecer a ideia de aleatoriedade. Uma amostra aleatória é obtida pela aplicação de uma das técnicas de amostragem, seja ela aleatória simples, estratificada, por conglomerados ou sistemática. Nas pesquisas 
quantitativas, as chamadas amostras de conveniência não respondem à questão da pesquisa de forma cientificamente correta e não produzem resultados confiáveis; portanto, na falta do modelo, não se aplica a estatística para o fim proposto.

Para executar testes estatísticos, é preciso conhecer os objetivos da pesquisa. Os objetivos são explicitações sobre o que o pesquisador quer fazer. Os objetivos primários são declarações generalizadas, ao passo que os objetivos secundários são declarações específicas. Caso o trabalho tenha hipóteses, elas devem ser descritas em forma testável. Por forma testável entende-se a forma na qual a hipótese pode ser falseada. Quando a pesquisa contiver teste de hipótese, a análise estatística será direcionada para aceitar ou rejeitar a hipótese e para identificar variáveis que, de alguma forma, interferem na relação estudada. Se não houver hipótese, as análises estatísticas serão realizadas de acordo com as explicitações dos objetivos.

Nos estudos analíticos, as hipóteses e objetivos de qualquer pesquisa são traduzidos para variáveis, as quais representam as ligações da dimensão teórica com a matemática. Em outras palavras, são os números obtidos por medições ou contagens que serão utilizados pelas fórmulas estatísticas. Quando se quer conhecer o tempo de vida de uma pessoa, escolhem-se a escala e a unidade de medida. Para uma pessoa adulta, a unidade de medida mais frequentemente usada são os anos. Se há necessidade de maior precisão, escolhem-se meses, semanas ou dias. Mas, quando se trata do momento do nascimento de uma criança, é preferível escolher a unidade em minutos ou horas. No primeiro mês de vida, é preferível a contagem em dias. Desse modo, a variável será, ora medida em anos, ora em meses, ora em semanas... Os testes estatísticos se utilizam de variáveis e são criados em conformidade com a escala de medidas, da qual se originam os testes paramétricos ou não paramétricos.

A estatística emprega contagens ou medições, o que exige precisão. Os termos indefinidos precisam ser objetivados de forma que possam ser mensurados (por exemplo, trabalhos de perfis ou qualidade precisam ser traduzidos para a forma mensurável). Parece que todos entendem o que significa perfil nutricional ou qualidade de vida. Entretanto, quando comparados, vê-se que os instrumentos de medidas foram diferentes e, consequentemente, não medem as mesmas dimensões. Para a ciência, a comunicação é essencial e, consequentemente, as ambiguidades devem ser eliminadas, especialmente nos casos dos modelos estatísticos que operam por generalização.
As variáveis compostas são geralmente conceituadas e obtidas por meio de questionários. Os conceitos devem ser bem estabelecidos e reconhecidos pela comunidade científica. Assim, por exemplo, é pertinente que uma pesquisa sobre qualidade de vida faça uso do questionário de qualidade de vida da Organização Mundial da Saúde, WHOQOL abreviado ${ }^{32}$, uma vez que esse já foi definido, testado, validado para a língua portuguesa e mundialmente aceito. Um novo instrumento de medida necessita passar por um processo de validação antes de ser aplicado.

Um teste de hipótese deve ter uma variável dependente e uma ou mais variáveis independentes, ou explicativas. Por exemplo: queremos testar a hipótese de que o tabagismo causa câncer de pulmão. Parece haver sentido nessa relação tabagismo-câncer. A variável independente é o tabagismo e a dependente - consequência do tabagismo - é o câncer. Pode-se testar mais de uma variável independente, como o asbesto e tabagismo, que potencializam seus efeitos, e outras mais.

No entanto, para assegurar a reprodutibilidade do estudo - característica essencial de qualquer projeto científico -, é necessário indicar com antecedência as variáveis que serão analisadas e estabelecer o vínculo teórico dessas variáveis com a revisão literária sistemática apresentada na introdução do trabalho. A violação desse item direciona as análises para os erros da aleatoriedade. Em trabalhos que envolvem relações entre variáveis, sempre haverá testes de hipóteses. Considerando o erro alfa de $5 \%$ (ou $p=0,05$ ), a cada 20 testes espera-se uma associação falso-positiva aleatória. Esse erro, de "cruzar os dados", pode ser obtido com a análise combinatória de 7 variáveis, resultando em 21 combinações.

A hipótese existe para ser testada, e é imperativo não esquecer que os dados jamais podem ser moldados para confirmá-la. A exploração de dados à procura de $p$ significativos que se adaptem a hipóteses é um caminho que não deve ser aceito. Não explicitar as variáveis que orientaram a análise estatística é como fazer um teste de proficiência com um atirador que não tem de antemão os alvos definidos. Imagine três tiros desferidos na direção de uma parede branca, sem qualquer alvo traçado. Depois de identificados os locais da parede atingidos pelos projéteis, desenham-se alvos ao redor deles, concluindo-se assim que os tiros foram certeiros.

Seguindo a mesma lógica, o pesquisador deve explicitar a priori os erros que aceita em seu estudo. $\mathrm{O}$ erro alfa está relacionado com o grau de proba- 
bilidade de chegar a uma explicitação falso-positiva e depende de uma decisão teórica do pesquisador. Já o erro beta determina o tamanho da amostra, e é importante estabelecê-lo para não tornar o trabaIho inconclusivo, seja por uma amostra pequena, podendo expor os participantes da pesquisa, seja por uma amostra de tamanho excessivo, o que produziria dados desnecessários ${ }^{6}$. Para o cálculo do tamanho da amostra, o pesquisador deve ter bem definida a questão da pesquisa e, no caso da análise multivariada, o número de variáveis que serão trabalhadas nas análises.

$\mathrm{Na}$ análise estatística de pesquisas médicas, importam outros fatores além desses, como a randomização, o uso de placebo e o cegamento. 0 equilíbrio (equipoise) ${ }^{33}$, obtido pela randomização, é um assunto ético de avaliação para os CEP, tratado na Resolução CNS 466/12, inciso III-2, alínea f:

...se houver necessidade de distribuição aleatória dos participantes da pesquisa em grupos experimentais e de controle, assegurar que, a priori, não seja possível estabelecer as vantagens de um procedimento sobre outro, mediante revisão de literatura, métodos observacionais ou métodos que não envolvam seres humanos. $O$ inciso III-3, alínea $b$, trata do placebo: ter plenamente justificadas, quando for o caso, a utilização de placebo, em termos de não maleficência e de necessidade metodológica, sendo que os benefícios, riscos, dificuldades e efetividade de um novo método terapêutico devem ser testados, comparando-o com os melhores métodos profiláticos, diagnósticos e terapêuticos atuais. Isso não exclui o uso de placebo ou nenhum tratamento em estudos nos quais não existam métodos provados de profilaxia, diagnóstico ou tratamento ${ }^{29}$.

Portanto, ter presentes os pressupostos da pesquisa é fundamental para o início das aplicações dos testes estatísticos e, se esses pressupostos não forem atendidos, a análise estatística não se aplicará ou não será confiável.

Fragilidades estatísticas que produzem resultados incorretos

A análise estatística pode obter resultados diferentes, dependendo da forma de análise dos dados empregada. Para decidir entre um teste paramétrico e um teste não paramétrico em determinada análise com amostra de pequeno tamanho, é necessário verificar o pressuposto de distribuição normal na população. A evidência de que a distri- buição populacional é normal leva a escolha para o teste paramétrico e, quando não normal, para o teste não paramétrico.

De maneira geral, é possível afirmar que, quando os valores e os gráficos não são conclusivos, os testes estatísticos são de grande valia. Nesse caso, podem-se citar pelo menos seis testes possíveis - Anderson-Darling; Cramer-von Mises; Lilliefors (Kolmogorov-Smirnov); Pearson; Shapiro-Wilk; Shapiro-Francia ${ }^{34}$-, possibilitando ao estatístico a escolha do teste que melhor se adapta à situação. Ressalte-se que essas escolhas são brechas abertas para a tendenciosidade. Assim como esse, outros exemplos poderiam ser citados.

O tratamento das perdas e dos valores extremos pode interferir no resultado. Exemplo disso nas pesquisas em saúde são as perdas ao acaso e as perdas por recusa de continuar a pesquisa, que não devem ser tratadas igualmente. A perda por recusa constitui um grupo à parte, que necessita de investigação. Em pequenas amostras, os valores extremos podem influenciar os resultados, especialmente nos testes de regressão. Em testes de hipóteses, cabe ao profissional justificar se esses valores serão excluídos ou se serão utilizados métodos não paramétricos. 0 tratamento das perdas e dos valores extremos está relacionado com o método estatístico empregado, o qual depende de decisões pessoais que só podem ser avaliadas com os resultados finais.

Também contribui para enganos a escolha do teste adequado a cada tipo de verificação e amostra. Tais equívocos podem decorrer da não verificação dos pressupostos da amostra ${ }^{4}$, como, por exemplo, aleatoriedade, distribuição normal da população, igualdade da variância, análise de resíduos. Podem estar relacionados com o emprego de escala de variável inadequada, tratando uma variável ordinal como se fosse nominal ou uma variável discreta como se fosse da razão, ou, ainda, com a variedade de resultantes na tela. Nesse último caso, por exemplo, é comum que a aplicação de um teste qui-quadrado produza na mesma tela o teste de Pearson, a correção de Yates e o teste de Fisher ${ }^{2}$. Cada um desses resultados se enquadra em uma situação especial ${ }^{35}$. Disso se depreende que a exploração de dados à procura de $p$ significativos que se adaptem a hipóteses é um caminho que não deve ser aceito.

Por fim, entre as fragilidades estatísticas capazes de produzir resultados incorretos, é preciso citar a área de atuação e o grau de experiência do estatístico. A estatística, cujos princípios são amplos, abrange diversas técnicas e áreas de atuação. Assim, por exemplo, um controle estatístico de qualidade 
aplicado na indústria emprega testes diferentes daqueles utilizados em ensaios clínicos, além de exigir capacitações profissionais diferentes. Por isso, para analisar projetos de pesquisa na área da saúde, não basta ser estatístico com formação somente em matemática; é necessário ampliar sua formação para a área da saúde.

Nesse sentido, cabe definir estatístico como a pessoa que realiza análises estatísticas de um projeto. Teremos, então, estatísticos ocasionais, que conhecem os conceitos básicos da área, mas que raramente analisam dados, e estatísticos experientes, capacitados por cursos especializados de epidemiologia e estatística e que se dedicam ao estudo e ao emprego da estatística na área da saúde. Os programas de estatística disponibilizados comercialmente para computadores permitem que as pessoas com pouca experiência analisem dados estatísticos. Basta ter uma planilha com dados, que os computadores irão produzir resultados.

Uma análise realizada sem a compreensão profunda dos fundamentos da estatística torna-se preocupante. Curran-Everett, Taylor e Kafadar ${ }^{36}$ criticam a abordagem da estatística em cursos de graduação e escolas médicas que enfatizam métodos e negligenciam o ensino dos fundamentos básicos da área. No artigo Fundamental concepts in statistics: elucidation and illustration, os autores revisam os fundamentos da estatística e abordam temas como estimativas de incertezas sobre parâmetros populacionais, significância estatística e científica e limitações da estatística. Escrito de forma objetiva e sintética, é recomendado àqueles que querem revisar ou compreender melhor o significado das fórmulas estatísticas e suas aplicações.

\section{Aspectos éticos}

A Resolução CNS 466/12, no inciso III-2, adverte: As pesquisas, em qualquer área do conhecimento envolvendo seres humanos, deverão observar as seguintes exigências: a) ser adequada aos princípios científicos que a justifiquem e com possibilidades concretas de responder a incertezas ${ }^{29}$. É nesse pressuposto ético que se apoiará o rigor do método científico, bem como as análises estatísticas.

Os testes de hipóteses carregam consigo um processo complexo de desenvolvimento que não admite erro. $O$ teste da hipótese nula deve resultar em uma de duas palavras antagônicas: aceita ou rejeita. Essas palavras indicam que uma teoria se opõe a outra e que somente uma delas repre- senta a verdade científica aceita no momento. No entanto, quando o tamanho da amostra é pequeno, o teste não indica resultado preciso, isto é, não rejeita, mas também não pode aceitar, o que torna impossível concluir. Tal situação adversa pode ser evitada com o cálculo adequado a priori do tamanho da amostra.

Todavia, se o tamanho da amostra não pode ser determinado, não é ético realizar o trabalho, pois implicaria submeter seres humanos a riscos desnecessários. E é justamente por causa dessa preocupação que a análise do CEP deve, de fato, ser rígida no sentido de verificar se todas as exigências ético-normativas estarão sendo atendidas por determinada pesquisa. Isso porque o CEP é corresponsável ético pela pesquisa, e qualquer falha não identificada no estudo recai sobre o comitê, seja por ter sido incapaz de identificar inconsistências metodológicas em um estudo, seja por aprovar um trabalho que não apresenta condições de responder à questão da pesquisa.

Ao aprovar um projeto, o CEP declara ao pesquisador e à comunidade científica a conformidade do trabalho com as normas que definem o que é eticamente admissível em estudos realizados no país. Dessa forma, o comitê assume a responsabilidade de ter zelado para que somente os erros inevitáveis aconteçam, certificando que todo o esforço foi realizado para que a população-alvo não sofra danos.

No Brasil, a Resolução CNS 466/12, em seu inciso III-3, determina que as pesquisas devem utilizar os métodos adequados para responder às questões estudadas, especificando-os, seja a pesquisa qualitativa, quantitativa ou quali-quantitativa ${ }^{29}$. Além de classificar os tipos de pesquisa, a norma confere aos CEP a responsabilidade de identificar o método adequado para responder à questão que orienta o estudo. Nesse contexto, torna-se fundamental delegar aos CEP a incumbência de analisar o desenho da pesquisa, considerando especialmente os parâmetros estatísticos, já que é a ferramenta analítica básica da pesquisa quantitativa.

Há projetos de pesquisa que descrevem ambiguamente a análise estatística. Vejamos um exemplo. As variáveis quantitativas serão testadas por meio de testes paramétricos ou, quando não aplicável, por testes não paramétricos. Os testes qui-quadrado serão utilizados para as variáveis qualitativas. O erro alfa esperado é $5 \%$. O programa estatístico será o SPSS 20.0. Sua descrição nada diz de importante. É como se o pesquisador estivesse solicitando ao CEP um cheque não preenchido e as- 
sinado, para que posteriormente os valores fossem completados com o produto disponível.

A seguir, exemplificamos a descrição estatística objetiva, supondo o teste de hipótese sobre tabagismo como fator de risco para o câncer de pulmão. Uma descrição estatística objetiva seria algo semelhante a: o câncer de pulmão será a variável dependente e o tabagismo, a variável independente. Será utilizado o teste qui-quadrado para testar a independência dos dados. A relação entre fumar e ter câncer por fumar e não ter câncer será feita por razões de chances (odds ratio). Conforme o delineamento da pesquisa há necessidade de ajustes de outras variáveis: os ajustes serão feitos por meio de regressão logística com as variáveis alcoolismo, exposição ao asbesto e gênero. Nesse caso, as variáveis incluídas na análise foram citadas nos objetivos secundários. A objetividade da descrição estatística demonstra quanto o pesquisador está situado na hipótese que está pesquisando. A escolha das variáveis foi resultado de estudo minucioso da literatura e foram incluídas no estudo por fazerem parte de uma teoria desenvolvida pelo pesquisador.

Vamos exemplificar a descrição estatística subjetiva dos testes estatísticos com as mesmas variáveis do exemplo anterior, mas com acréscimo de outras que poderiam constituir um resultado interessante. Agora o estatístico utilizará o câncer de pulmão como variável dependente e fará análises bivariadas de todas as outras variáveis como variáveis independentes. A partir dos resultados, irá formular a hipótese, pois as variáveis que resultarem em $p<$ 0,05 se tornarão fator de risco. Nesse exemplo, as variáveis foram listadas com a expectativa de que alguma resulte em $p<0,05$.

O erro alfa propicia que o acréscimo do número de variáveis analisadas aumente a probabilidade de que alguma resulte no $p$ esperado. Esse estudo não tem o poder de concluir sobre fator de risco, pois não passa de um estudo descritivo, e o máximo que pode produzir é uma hipótese que necessitará de outro estudo específico para testá-la. Nesse modelo, chamado de "cruzamento de dados", o pesquisador terá liberdade de escolher as variáveis que julga mais interessantes para elaborar o artigo que irá submeter à publicação, o que constitui viés de estatística. Assim, se o texto for aprovado, qualquer resultado terá o aval ético do CEP. O trabalho de Haas ${ }^{37}$ aborda mais profundamente esse assunto.

Os CEP têm limitações que os impossibilitam de analisar os dados. Desse modo, não há como identificar se as análises foram avaliadas de acordo com os pressupostos dos testes e a escolha foi adequa- da. Mas os CEP têm poderes para julgar a adequação do planejamento do projeto de pesquisa e verificar, no final, se o executado correspondeu ao planejado. Estudos de May ${ }^{1}$, em 1975, e Altman ${ }^{9}$, em 1981, mostram a importância da estrutura do projeto de pesquisa para a ética, defendendo a presença de estatísticos experientes nos CEP.

May se baseia no princípio de que a garantia primária de proteção dos sujeitos de pesquisa contra riscos desnecessários e abusos acontece na revisão, antes de a pesquisa começar. Assim, a aprovação do projeto de pesquisa depende da aprovação da descrição estatística. Em 1994, treze anos após a publicação do trabalho de Altman ${ }^{9}$, verificou-se que a falta de julgamento estatístico das pesquisas continuava acontecendo na Inglaterra.

No mesmo ano, o próprio Altman ${ }^{38}$, em editorial publicado no British Medical Journal com o título "The scandal of poor medical research", voltava a abordar o assunto. No entanto, só 10 anos mais tarde, em 2004, o estatístico foi incluído na regulamentação para a composição dos comitês de ética em pesquisas no Reino Unido pelo National Research Ethics Service (NRES) ${ }^{39,40}$.

Por outro lado, no Brasil, a Resolução CNS 466/12 parece contemplar a análise estatística na alínea 9 do inciso X-3: uma vez aprovado o projeto, $O$ CEP, ou a Conep, na hipótese em que atua como CEP ou no exercício de sua competência originária, passa a ser corresponsável no que se refere aos aspectos éticos da pesquisa ${ }^{29}$. Depreende-se, desse texto, que os CEP devem reunir argumentos suficientes para justificar a aprovação dos projetos de pesquisa e, com isso, sua responsabilidade se iguala à do pesquisador no que concerne às análises estatísticas citadas no projeto. Decorre a conclusão lógica de que a falha do CEP em reunir argumentos para defender o projeto de pesquisa implica a não aprovação do projeto. No entanto, é autorizado o funcionamento de comitês que não dispõem de conselheiros estatísticos, o que pode prejudicar a devida avaliação e aprovação do projeto de pesquisa.

Por outro lado, como a atividade dos participantes dos CEP é voluntária, dificilmente se conseguiria que todos os comitês contassem com estatísticos experientes para avaliar todos os projetos de pesquisa, mesmo tendo em vista o caráter multidisciplinar previsto em sua composição. A dificuldade de contar com estatísticos nos CEP, bem como a possível sobrecarga de trabalho desses profissionais, uma vez que são os mais bem capacitados para analisar aspectos essenciais de uma miríade de projetos, mostra que a discussão da proposta precisa 
ser aprofundada, a fim de que se possam encontrar soluções viáveis para todo sistema CEP/Conep.

Diante dessa dificuldade e do não estabelecimento de normas específicas para a análise estatística dos projetos de pesquisa contemplados na Resolução CNS 466/12 ${ }^{29}$, há comitês que acabam avaliando com maior ou menor rigor os estudos com testes estatísticos, o que abre a possibilidade, por exemplo, de agências de pesquisas e pesquisadores encaminharem os ensaios clínicos aos CEP mais flexíveis. Considerando tais problemas contingenciais, poderia ser útil estabelecer um roteiro para análise dos aspectos estatísticos dos projetos submetidos à avaliação ética. Assim, com intuito de diminuir diferenças entre os CEP quanto aos critérios de avaliações, propõe-se um roteiro com orientações básicas acerca dos itens necessários à análise da estatística em projetos de pesquisa quantitativos, conforme descritos na Tabela 1.

Tabela 1. Itens básicos necessários às análises estatísticas em projetos de pesquisa

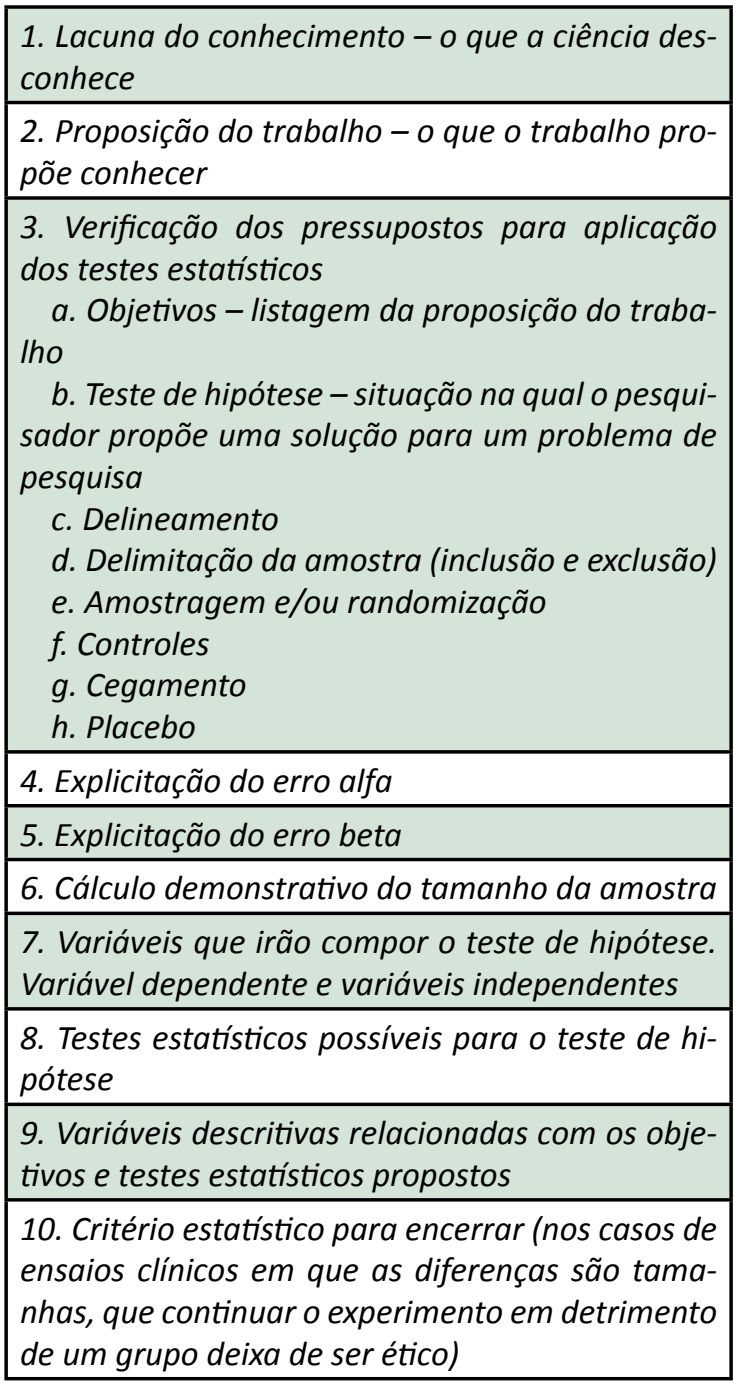

Ademais, no que tange à responsabilidade ética dos CEP, é importante considerar que, assim como a Conep, os comitês são instâncias cujo poder emana diretamente do CNS, o mais alto fórum do controle social da saúde. Por conseguinte, a responsabilidade ética dos CEP e da Conep na apreciação dos protocolos de pesquisa extrapola a dimensão normativa, revestindo-se do caráter ético que move o espírito democrático modelador da Constituição Federal.

Além das instâncias do sistema CEP/Conep, também é preciso levar em conta que a responsabilidade ética pelos estudos é partilhada a posteriori pelos periódicos científicos, cujos revisores avaliam o recorte metodológico das pesquisas relatadas em artigos científicos submetidos à publicação. Em geral, os periódicos adotam regras rígidas na análise do método científico dos trabalhos, uma vez que sua credibilidade está diretamente relacionada com esse rigor. Assim, cumprem o papel de guardiões da qualidade dos estudos ${ }^{9}$.

Cabe notar, porém, que, mesmo sendo frustrante para o pesquisador verificar que seu projeto foi rejeitado pelas revistas científicas por falha metodológica, a constatação de irregularidade pelos CEP, na fase de projeto, permite prevenir os efeitos da exposição dos participantes aos riscos do experimento, já que as publicações científicas nada podem fazer para proteger o sujeito da pesquisa. Nessa fase, a melhor forma de avaliação da experiência e do rigor estatístico parece ser a objetividade da descrição estatística.

Assim, quanto mais objetiva e detalhada a descrição, mais precisa poderá ser a análise. Se não existir detalhamento descritivo, qualquer resultado será possível. No que tange às garantias aos participantes, é preferível o CEP detectar erros metodológicos na fase de projeto, evitando assim danos aos sujeitos de pesquisas, a ter os trabalhos não publicados pelas revistas sob a alegação de erros metodológicos. Nesse caso, além de rechaçar o trabalho, cabe reprovação adicional à equipe que realizou a pesquisa, com extensão aos avaliadores do sistema CEP/Conep, porque, conforme o inciso VII-4 da Resolução CNS 466/2012: A revisão ética dos projetos de pesquisa envolvendo seres humanos deverá ser associada à sua análise científica ${ }^{29}$.

Há que mencionar a ideia promissora da pesquisa reprodutível, desenvolvida por Christopher Gandrud no livro Reproducible research with $\mathrm{R}$ and RStudio, ${ }^{41}$ que pode resultar em solução melhor que a proposta neste artigo; porém, haverá longo caminho a ser percorrido até que seja aceita pela comunidade 
científica. Esse autor compara as publicações dos trabalhos científicos atuais com propagandas. Para ele, a pesquisa consiste na disponibilização aos pesquisadores independentes do ambiente computacional, do código e dos dados que produziram os resultados, de forma que seja possível reproduzir o trabalho e aprimorá-lo. Atualmente, existem ferramentas que tornam essa ideia factível, como o programa $\mathrm{R}^{42}$, utilizado em grandes universidades e por pesquisadores de todo o mundo, juntamente com o RStudio ${ }^{43}$, que incorpora recursos para facilitar o manuseio do $\mathrm{R}$, as linguagens LaTeX ${ }^{44}$ ou Markdown ${ }^{45}$, com o package knitr ${ }^{46}$, todos disponibilizados gratuitamente pela Internet. No entanto, problemas éticos e de direitos autorais ainda precisam ser discutidos e normatizados antes de ser implantada.

Pesquisas reprodutivas conforme o modelo exposto evitariam boa parte dos problemas discutidos neste artigo, e seriam capazes de identificar situações éticas ou de armadilha estatística impossíveis de ser detectadas sem reanálises. Quando a análise estatística não é mostrada o resultado se torna uma questão de fé, no entanto, sendo apresentada se torna uma questão técnica. Como exemplo da questão ética sobre teste de regressão múltipla, destacamos o capítulo 15.5 do livro "Estatística: teoria e aplicações - usando o Microsoft Excel", de Levine, Stephan, Krehbiel e Berenson:

...há um comportamento fora dos padrões éticos quando alguém utiliza a análise de regressão múltipla e intencionalmente deixa de remover da avaliação variáveis que apresentam uma elevada colinearidade com outras variáveis independentes, ou intencionalmente deixa de utilizar métodos outros que não a regressão dos mínimos quadrados quando os pressupostos necessários para a regressão dos mínimos quadrados são seriamente violados ${ }^{47}$.

A disponibilização dos dados e códigos de análises como proposto nas pesquisas reprodutivas seria um grande avanço para a ciência, mas não resolveria a questão da falsificação dos dados brutos. Todavia, a transparência para com a comunidade científica junto com a publicação seria um ato ético de respeito aos seres humanos e diminuiria o peso que hoje têm o CEP e as revistas científicas, mas sem eliminar suas responsabilidades.

\section{Considerações finais}

Sendo a estatística um componente da pesquisa, há que admitir sua relação com a ética. É considerado eticamente adequado o projeto de pesquisa completo, objetivo, que responde às questões de pesquisas quando aprovado por um comitê de ética competente. O erro resultante de uma pesquisa científica adequada somente se justifica eticamente quando o método científico foi aprovado a priori e executado em sua totalidade e integridade.

O método científico aplicado aos estudos quantitativos em seres humanos utiliza a teoria da probabilidade por meio da estatística. Nesses trabaIhos, a estatística é instrumento vital para a aplicação da ética em pesquisa, especialmente nos ensaios clínicos, por sua potencialidade de produção de dano. Por conseguinte, não há como separar, no âmbito dos CEP, a análise ética da estatística nos projetos de pesquisa quantitativos. Descrições estatísticas incompletas, equivocadas ou ambíguas não atendem às exigências de aprovação. É importante, então, que os CEP, assim como a Conep, não deixem de proceder à adequada avaliação dos aspectos estatísticos dos estudos.

$\mathrm{O}$ instrumento de controle de um projeto de pesquisa estatisticamente bem elaborado pode ser diferente do proposto neste trabalho, mas fiscalização e monitoramento são necessários e precisam ser aplicados. Caso contrário, a normatização ética da pesquisa no Brasil não alcançará integralmente o seu objetivo.

\section{Agradecimentos}

Agradeço à Dra. Ana Maria Camargo, Dra. Ana Paula Delamare, Dr. Antonio Bangel, Sra. Glaucia Gomes, Sra. Isoldi Chies, Dr. José Ferreira Machado, Dra. Luciane Bizzi, Dra. Maria Helena Wagner Rossi, Dra. Margharet Borella, Dra. Marlene Branca Solio, Dr. Paulo Cesar Nodari e Dr. Petronio Fagundes de Oliveira Filho, pelas numerosas horas de discussões éticas de análises de projetos de pesquisa. Agradeço ao Dr. André Leite, Dr. Clayton de Macedo e Dra. Liliana Zugno Filippini Spiandorello, pela revisão final do texto.

\section{Referências}

1. May WW. The composition and function of ethical committees. J Med Ethics. 1975 fev;1(1):23-9. 
2. Altman DG. Statistics and ethics in medical research: VII-Interpreting results. $\mathrm{Br}$ Med J. 1980;281(6.255):1.612-4.

3. Altman DG. Statistics and ethics in medical research: VI-Presentation of results. Br Med J. 1980;281(6.254):1.542-4.

4. Altman DG. Statistics and ethics in medical research: V-Analysing data. $\mathrm{Br}$ Med J. 1980;281(6.253):1.473-5.

5. Altman DG. Statistics and ethics in medical research: collecting and screening data. Br Med J. 1980;281(6.252):1.399-401.

6. Altman DG. Statistics and ethics in medical research: III-How large a sample? $\mathrm{Br}$ Med J. 1980;281(6.251):1.336-8.

7. Altman DG. Statistics and ethics in medical research: study design. $\mathrm{Br}$ Med J. 1980;281(6.250):1.267-9.

8. Altman DG. Statistics and ethics in medical research: misuse of statistics is unethical. $\mathrm{Br}$ Med J. 1980;281(6.249):1.182-4.

9. Altman DG. Statistics and ethics in medical research: VIII-Improving the quality of statistics in medical journals. Br Med J (Clin Res Ed). 1981;282(6.257):44-7.

10. Gilbert JP, McPeek B, Mosteller F. Statistics and ethics in surgery and anesthesia. Science. 1977;198(4.318):684-9.

11. Rathnam A. TAPVC vital statistics: ethics and moral values. Am Heart J. 1977;93(5):676.

12. Clarke MR. Statistics and ethics in medical research. Br Med J (Clin Res Ed). 1981;282(6.262):480.

13. Dobbing J. Statistics and ethics in medical research. Br Med J (Clin Res Ed). 1981;282(6.268):990.

14. Zander LI. The place of confinement: a question of statistics or ethics?. J Med Ethics. 1981;7(3):125-7.

15. Kulcar Z, Hrabak-Zerjavic V. [The ethics of medical statistics]. Lijec Vjesn. 1983;105(11-12):488-90.

16. Yates FE. Contribution of statistics to ethics of science. Am J Physiol. 1983 ;244(1):R3-5.

17. Browner WS. Ethics, statistics, and technology assessment: the use of a stopping rule and an independent policy and data monitoring board in a cohort study of perioperative cardiac morbidity. The study of Perioperative Ischemia Research Group. Clin Res. 1991;39(1):7-12.

18. Royall RM, Bartlett RH, Cornell RG, Byar DP, Dupont WD, Levine RJ et al. Ethics and statistics in randomized clinical trials. Stat Sci. 1991;6(1):52-88.

19. Anez MS, Canelon FM. [Research, publications, statistics and ethics]. G E N. 1992;46(4):328-30.

20. Perel ML. Ethics and the three faces of statistics. Implant Dent. 1998;7(4):249-50.

21. DeMets DL. Statistics and ethics in medical research. Sci Eng Ethics. 1999 jan;5(1):97-117.

22. Gasko R. [Discussion of the article by T. Haas "Ethics and statistics - difficult approaches to data"]. Cas Lek Cesk. 2000;139(19):607

23. Haas T. [Ethics and statistics: difficult approaches to data]. Cas Lek Cesk. 2000;139(3):88-94.

24. Upshur RE. The ethics of alpha: reflections on statistics, evidence and values in medicine. Theor Med Bioeth. 2001;22(6):565-76.

25. Scheen AJ. Clinical study of the month. Premature interruption of ASCOT and CARDS clinical trials of cardiovascular prevention with atorvastatin in patients with arterial hypertension or diabetes mellitus: compromise between ethics and statistics in evidence-based medicine. Rev Med Liege. 2003;58(9):585-90.

26. Cassel CK. Statistics and ethics: models for strengthening protection of human subjects in clinical research. Proc Natl Acad Sci U S A. 2009;106(52):22.037-8.

27. Pitak-Arnnop P. Statistics or ethics? Decision to treat drooling. Arch Otolaryngol Head Neck Surg. 2010;136(3):315.

28. Abhaya I. Basic of medical studies. In: Press C, editor. Medical biostatistics. 3a ed. Londres: Chapman \& Hall/CRC Biostatistics Series; 2013. p. 54-61.

29. Conselho Nacional de Saúde. Resolução no 466, de 12 de dezembro de 2012. Aprova as diretrizes e normas regulamentadoras de pesquisas envolvendo seres humanos. [Internet]. CNS; 2012 [acesso 20 out 2013]. Disponível: http://conselho.saude.gov.br/resolucoes/2012/Reso466.pdf

30. Hochman B, Nahas FX, Oliveira Filho RS de, Ferreira LM. Desenhos de pesquisa. Acta Cir Bras. [Internet]. 2005[acesso nov 2014];20(Supl. 2):2-9. Disponível: http://dx.doi.org/10.1590/S010286502005000800002

31. Upton G, Cook I. Oxford dictionary of statistics. $2^{\text {nd }}$ ed. New York: Oxford University Press; 2006. Statistics; p. 408.

32. Organização Mundial da Saúde. Divisão de Saúde Mental. Grupo WHOQOL no Brasil. WHOQOL - abreviado. In: Fleck MPA, coordenador. Instrumentos de avaliação da qualidade: versão em português. [Internet]. Porto Alegre: Universidade Federal do Rio Grande do Sul; 1998 [acesso 1응 nov 2013]. Disponível: http://www.ufrgs.br/psiquiatria/psiq/breve.PDF

33. Cassel CK. Statistics and ethics: models for strengthening protection of human subjects in clinical research. Proc Natl Acad Sci U S A. 2009;106(52):22.037-8.

34. Gross J, Ligges U. Nortest: tests for normality version 1.0-2. [Internet]. 2012 [acesso 1으 nov 2013]. Disponível: http://CRAN.R-project.org/package=nortest

35. Gore SM, Jones IG, Rytter EC. Misuse of statistical methods: critical assessment of articles in BMJ from January to March 1976. Br Med J. 1977;1:85-7.

36. Curran-Everett D, Taylor S, Kafadar K. Fundamental concepts in statistics: elucidation and illustration. Journal of Applied Physiology. 1998;85(3):775-86. 
37. Haas T. [Ethics and statistics: difficult approaches to data]. Cas Lek Cesk. 2000;139(3):88-94.

38. Altman DG. The scandal of poor medical research. Br Med J. [Internet]. 1994 [acesso nov 2014];308(6.924):283-4. Disponível: http://www.ncbi.nlm.nih.gov/pmc/articles/PMC2539276/ pdf/bmj00425-0005.pdf

39. National Research Ethics Service. Health Research Authority. [Internet]. London: NHS/HRA; [acesso 15 ago 2014]. Disponível: http://www.nres.nhs.uk

40. RECs in the News. 2009 jun;(57). Disponível: http://www.nres.nhs.uk/search/?q=statistics

41. Gandrud C. Reproducible research with R and RStudio. Boca Raton: CRC Press; 2014. p. 3-16.

42. R Foundation for Statistical Computing. The R Project for Statistical Computing. [Internet]. Version 3.0.3. 6 mar 2014 [acesso 15 ago 2014]. Disponível: http://www.r-project.org

43. RStudio Inc. RStudio. [Internet]. Boston: RStudio; 2014 [acesso 15 ago 2014]. Disponível: http:// www.rstudio.com

44. LaTeX Project. LaTeX: a document preparation system. [Internet]. 2008 [acesso 15 ago 2014]. Disponível: http://www.latex-project.org

45. Fireball D. Markdown. [Internet]. Version 1.0.1. 17 feb 2004 [acesso 15 ago 2014]. Disponível: http://daringfireball.net/projects/markdown

46. Yihui Xie. Knitr: elegant, flexible and fast dynamic report generation with R. [Internet]. 2011 [acesso 15 ago 2014]. Disponível: http://yihui.name/knitr

47. Levine DM, Stephan DF, Krehbiel TC, Berenson ML. Estatística: teoria e aplicações. Usando o Microsoft Excel em português. 6ạ ed. Rio de Janeiro: Gen/LTC; 2012. p. 604.

Recebido: 19.11 .2013

Revisado: 18.3 .2014

Aprovado: 18.10 .2014 